\title{
Le plan stratégique est mort, vive le plan culturel!
}

\author{
par Clarence Chant
}

Selon le dictionnaire Larousse, la culture est un « ensemble des $\checkmark$ phénomènes matériels et idéologiques qui caractérisent un groupe ethnique ou une nation, une civilisation, par opposition à un autre groupe ou à une autre nation $»^{1}$. Cette définition s'applique aussi aux microsociétés que sont les groupes de professionnels (notamment les pharmaciens), les établissements où ceux-ci travaillent et même au Journal canadien de la pharmacie hospitalière (JCPH), lui qui n'est « jamais fermé et en constante rénovation $n^{2}$ ! Dernièrement, dans les différents programmes de formation en pharmacie du pays, l'accent a été mis sur la sensibilisation aux diverses origines et cultures des patients ainsi que sur l'enseignement des moyens pour prendre en compte ces caractéristiques afin de devenir de meilleurs praticiens. Toutefois, l'importance de comprendre la culture des pharmaciens comme groupe distinct n'a pas encore réussi à bien s'ancrer, que ce soit dans les programmes de premier cycle ou les cours de perfectionnement professionnel. Des exemples tirés du secteur privé, où l'expression « culture eats strategy for breakfast (la culture ne fait qu'une bouchée de la stratégie) » est devenue à la mode, peuvent permettre de voir en quoi il est nécessaire de comprendre notre propre culture (ainsi que celle des membres de notre équipe, soit les médecins et le personnel infirmier). Attribuée à Peter Drucker, un conseiller américain en gestion, cette expression a été adoptée par bon nombre d'entreprises, dont Ford Motor Company'. Selon certains, c'est une façon de dire que peu importe qu'un organisme offre une définition claire de sa mission et de son plan stratégique, leur mise en œuvre échouera à moins que la mission et le plan ne soient accompagnés d'une bonne connaissance de la culture de l'organisation et qu'ils y soient conformes. Autrement dit une entreprise aura du succès, même au cours d'une période économique difficile et même lorsqu'elle rencontre des obstacles majeurs, si sa culture et ses valeurs sont clairement établies. En effet, des articles touchant au sujet laissent à penser que le rendement organisationnel dépendrait en fait de la culture ${ }^{4}$.
Plusieurs articles récents du JCPH ont abordé directement ou indirectement la notion de culture. Rosenthal et collab. ${ }^{5}$ ont cherché à étudier la culture professionnelle et les traits de personnalité des pharmaciens hospitaliers du Canada. Les auteurs ont employé l'outil normalisé de mesure de la culture organisationnelle, le "Organizational Culture Profile », dans le but de mieux connaitre la culture de la pharmacie hospitalière ainsi que l'outil sur les cinq traits centraux de la personnalité, le « Big Five Inventory ", afin de cerner les traits de personnalité des répondants. Comparativement aux données publiées antérieurement, les 401 pharmaciens hospitaliers ayant répondu au sondage ont accordé une plus grande importance aux facteurs de soutien, de compétitivité et de stabilité, contrairement à l'innovation, à la responsabilité sociale ainsi qu’à la motivation par la performance ou les récompenses. Lorsque le profil culturel et les traits de personnalité des répondants étaient corrélés à certaines " caractéristiques culturelles ", on semblait observer une interaction entre le niveau de scolarité et les domaines de l'innovation et de la compétitivité. Des liens entre certains aspects culturels et le nombre d'années de pratique de même que la région d'exercice ont aussi été constatés. De plus, l'enjeu concernant la place qu'occupe la culture actuelle des médias sociaux dans la pratique de la pharmacie a été abordé dans un autre article récent du $J C P H^{6}$. D'ailleurs, le JCPH publiera bientôt une série d'articles dressant un portrait des pratiques de la pharmacie de partout dans le monde, ce qui mettra sans doute en relief certaines différences culturelles importantes ainsi que leur influence sur la pratique.

Rosenthal et ses collègues méritent d'être félicités pour leur récent travail, qui vient s'ajouter à leur examen précédent sur la culture des pharmaciens communautaires ${ }^{7}$. Dans cette étude, ils avaient observé que la culture des pharmaciens communautaires était axée sur les produits et que les pharmaciens se considéraient principalement comme des « distributeurs de médicaments ». Le peu de documentation sur la culture de notre profession a été 
analysée par Jacobs et collab. ${ }^{8}$, qui n'ont trouvé qu’une poignée d'études portant sur la culture des pharmaciens, principalement sur celle des pharmaciens communautaires. Les récentes études, notamment celles mentionnées ci-dessus ${ }^{5-7}$, représentent d'importants premiers pas qui nous permettront de mieux nous connaître et ainsi de nous aider dans l'élaboration future de plans et de stratégies ayant comme objectif la modification des comportements des pharmaciens. Il est capital pour chacun d'entre nous, et pour les établissements où nous travaillons, de comprendre notre propre culture. Trop souvent, des leaders en pharmacie ont entrepris des changements qui avaient une portée sur les pharmaciens de première ligne (par exemple, la saisie électronique des ordonnances par les prescripteurs) sans avoir d'abord cherché à bien comprendre la culture, et leurs tentatives ont eu peu d'effets ou, pire encore, ont complètement échouées. Mais il n'y a pas que la culture du public cible qui soit importante, celle de l'initiateur de changement l'est tout autant parce que ses croyances et ses valeurs peuvent aussi contribuer au succès ou à l'échec de l'entreprise. Bon nombre d'entre nous avons dirigé des projets changeant la pratique qui ont connu un succès remarquable parce que, sans qu'on sache trop comment, la culture de la population ciblée s'est modifiée pour épouser l'intention du projet. Par exemple, dans l'ancienne culture des unités de soins intensifs (USI), l'hyperglycémie était à prévoir dans toutes maladies graves, et les cliniciens ne la jugeaient pas digne d'intérêt. Ainsi, la mise en place de moyens de régulation plus serrés de la glycémie aux USI n’a fonctionnée que lorsque les croyances du personnel infirmier ont été transformées; passant de "nous ne portons pas attention à la glycémie parce que l'hyperglycémie est normale et donc n'est pas un problème " à « il est prouvé que traiter l'hyperglycémie représente un facteur important pour le rétablissement de mes patients »!

Ainsi, dans la majorité des établissements et des services de pharmacie, la culture n'est ni connue ni définie, et peu d'efforts, voire aucun, sont faits pour la créer, la définir ou la transformer. Or, les pharmaciens, tout particulièrement ceux qui travaillent en milieu hospitalier, jouent souvent un rôle de dirigeant ou de participant dans les processus de changement à grande échelle, comme l'amélioration de la qualité et la planification stratégique. Donc, selon la prémisse voulant qu'une culture puisse être définie et, une fois cela fait, qu'elle puisse être modifiée, il semble que nous n'ayons que trop tardé à créer un plan culturel; surtout si nous souhaitons accroître les chances de succès de nos plans d'amélioration de la qualité et de nos plans stratégiques!

[Traduction par l'éditeur]

\section{References}

1. Le dictionnaire Larousse. Les Éditions Larousse; 2015. Publié au : www. larousse.fr/dictionnaires/francais/culture/21072?q=culture\#20950. Consulté le 13 mai 2015.

2. Ensom MHH. Quarante-cinq ans de JCPH : jamais fermé et en constante rénovation [éditorial]. Can J Hosp Pharm. 2014:67(6):408-9.

3. Simonelli M. Culture eats innovation for lunch. Innovation Excellence; 2014. Publié au : www.innovationexcellence.com/blog/2014/09/13/culture-eats-innovation-for-lunch/. Consulté le $1^{\text {er }}$ mars 2015.

4. Scott T, Mannion, R, Marshall M, Davies, H. Does organizational culture influence health care performance? A review of the evidence. J Health Serv Res Policy. 2003;8(2):105-17.

5. Rosenthal, MM, Hall K, Bussières JF, Tsuyuki R. Professional culture and personality traits of hospital pharmacists across Canada: a fundamental first step in developing effective knowledge translation strategies. Can J Hosp Pharm. 2015;68(2):127-35.

6. Barry AR, Pearson, GJ. Professional use of social media by pharmacists. Can J Hosp Pharm. 2015;68(1):22-7.

7. Rosenthal MM, Breault RR, Austin Z, Tsuyuki RT. Pharmacists' self-perception of their professional role: insights into community pharmacy culture. $J \mathrm{Am}$ Pharm Assoc. 2011;51:363-7.

8. Jacobs $\mathrm{S}$, Ashcroft $\mathrm{D}$, Hassell K. Culture in community pharmacy organizations: What can we glean from the literature? J Health Organ Manag. 2011;25(4): 420-54.

Clarence Chant, Pharm. D., BCPS, FCCP, FCSHP, est chef de pharmacie au St Michael's Hospital, à Toronto, en Ontario. II est aussi rédacteur adjoint du JCPH.

Intérêts concurrents : Aucun déclaré.

Adresse de correspondance :

Dr Clarence Chant

Pharmacy Department

St Michael's Hospital

30 Bond Street, Room B0007

Toronto ON M5B 1W8

Courriel: chantc@smh.ca 